\title{
Mixed convection in a three-dimensional ventilated cavity with two isothermal heating Sections
}

\author{
Hicham Doghmi ${ }^{1, *}$, Btissam Abourida ${ }^{1}$, Lahoucine Belarche ${ }^{1}$, Mohammed Sannad ${ }^{1}$, and \\ Meriem Ouzaouit ${ }^{1}$ \\ ${ }^{1}$ Ecole Nationale des Sciences Appliquées d'Agadir, BP 1136 Agadir, Maroc.
}

\begin{abstract}
Mixed convection in a three-dimensional ventilated cavity discretely heated has been analyzed numerically by the finite volume method. The results are presented in terms of streamlines, temperature distribution and average Nusselt number; for different combinations of thermal controlling parameters namely, Reynolds and Richardson numbers. The computational results indicate that the heat transfer's rate is strongly affected by the mentioned parameters.
\end{abstract}

\section{Introduction}

The study of the heat surplus evacuation problems has been the subject of extensive research for many years, due to their importance in electronic equipments, air-cooling process and design. The obtained results showed that mixed convection is the most simple and low-cost mode of cooling operation. In addition, this phenomenon is also involved in many engineering applications such as the thermal design of buildings, furnace design, air conditioning, nuclear reactors and others... This practical interest explains the existence of various studies such as the numerical approach of Papanicolaou \& Jaluria $[1,2]$ who studied the mixed convection in a ventilated rectangular cavity provided with a localized heat source. Their principal results showed that the average Nusselt number increases by increasing the solid wall thermal conductivity. The cooling rate was also found to be higher when the outlet flow opening was located near the bottom of the vertical wall. Hsu et al. [3] conducted a numerical study of the mixed convective flow inside an enclosure with a partially dividing partition. They concluded that the maximum heat transfer rate dissipated from the source is obtained when the outflow opening is located at the lower part of the vertical wall, and a better cooling system was designed by placing the heat source as close as possible from the cold stream inlet opening. Rahman et al. [4] investigated numerically the effects of Reynolds and Prandtl numbers on mixed convective flow and heat transfer characteristics in a ventilated cavity in presence of a heat-generating solid circular obstacle placed at the center. They indicated that the average Nusselt number at the heated surface for different values of Ri can be increased with the increasing of the Reynold and Prandtl

* Corresponding author: hicham.gte@gmail.com 
numbers. On the other hand, Moraga \& López [5] compared numerically the mixed convection in a two- and three-dimensional air-cooled cavity They found that a 3-D model must be used to capture the fluid mechanics for $\mathrm{Ri}=10$ when $10 \leq \mathrm{Re} \leq 250$, and to calculate the global Nusselt number when $\mathrm{Re}=500$ for $\mathrm{Ri}<1$.

Hence, the literature review shows that the majority of the previous works consider the case of two-dimensional model, while very few studies have been conducted for 3D numerical simulation. Therefore the three-dimensional approach gives a better modelization of the fluid flow and heat transfer within the cavity. This explains the choice of our present physical model which intended to investigate numerically the effects of conjugated Richardson and Reynolds numbers on the thermal transport and fluid flow phenomena in the considered cubical enclosure.

\section{Problem formulation}

The considered geometry is presented in Figure 1. It consists of a three-dimensional ventilated cavity with two heating square portions, similar to the integrated electronic components, are located on the vertical wall, while the rest of the considered wall is adiabatic. The opposite vertical wall is maintained at a cold uniform temperature, while the other four walls are adiabatic. An external air -flow enters the enclosure through rectangular cross section at the top of the left wall and exits from a channel, with the same cross section, placed at the bottom of the right vertical wall to extend the cavity and make possible the use of developed boundary conditions at the outlet for the fluid flow and heat transfer. In fact, many tests has been done in order to check the influence of the channel length on the outlet boundary conditions. Finally, a length of $\mathrm{L}$ has been adopted as it gives a good compromise between the results accuracy and the computational time.

The cooling flow is air having a Prandtl number Pr of 0.71 and is assumed to be laminar and incompressible. Viscous dissipation is negligibly small and all other fluid properties are assumed constant except the fluid density giving rise to the buoyancy forces (Boussinesq approximation).Under the above assumptions, the Governing equations of heat transfer and fluid flow can be expressed in three-dimensional dimensionless form as follows:

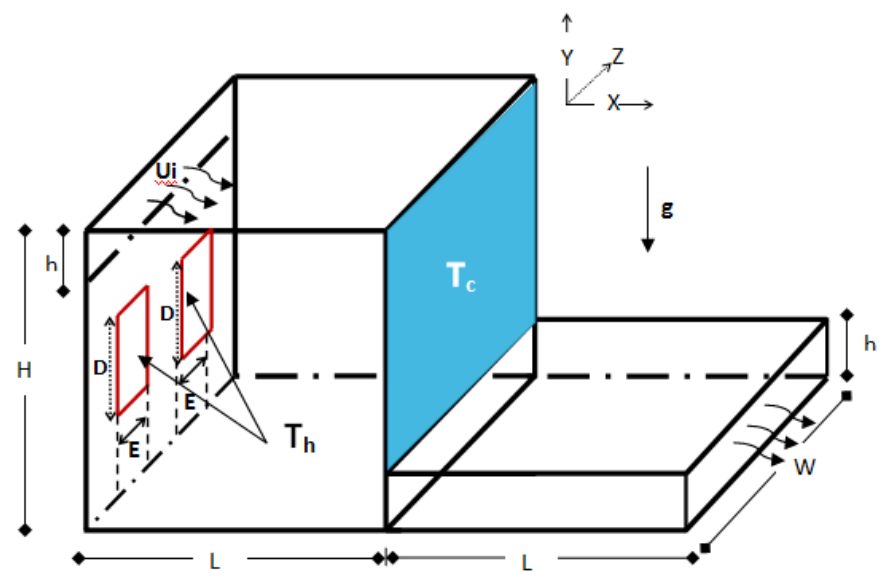

Fig. 1. Physical model and coordinate system.

$$
\frac{\partial U}{\partial X}+\frac{\partial V}{\partial Y}+\frac{\partial W}{\partial Z}=0
$$




$$
\begin{aligned}
& U \cdot \frac{\partial U}{\partial X}+V \cdot \frac{\partial U}{\partial Y}+W \cdot \frac{\partial U}{\partial Z}=-\frac{\partial P}{\partial X}+\frac{1}{\operatorname{Re}}\left(\frac{\partial^{2} U}{\partial X^{2}}+\frac{\partial^{2} U}{\partial Y^{2}}+\frac{\partial^{2} U}{\partial Z^{2}}\right) \\
& U \cdot \frac{\partial V}{\partial X}+V \cdot \frac{\partial V}{\partial Y}+W \cdot \frac{\partial V}{\partial Z}=-\frac{\partial P}{\partial Y}+\frac{1}{R e}\left(\frac{\partial^{2} V}{\partial X^{2}}+\frac{\partial^{2} V}{\partial Y^{2}}+\frac{\partial^{2} V}{\partial Z^{2}}\right)+\frac{G r}{R e^{2}} q \\
& U \cdot \frac{\partial W}{\partial X}+V \cdot \frac{\partial W}{\partial Y}+W \cdot \frac{\partial W}{\partial Z}=-\frac{\partial P}{\partial Z}+\frac{1}{R e}\left(\frac{\partial^{2} W}{\partial X^{2}}+\frac{\partial^{2} W}{\partial Y^{2}}+\frac{\partial^{2} W}{\partial Z^{2}}\right) \\
& U \cdot \frac{\partial \theta}{\partial X}+V \cdot \frac{\partial \theta}{\partial Y}+W \cdot \frac{\partial \theta}{\partial Z}=\frac{1}{\operatorname{RePr}}\left(\frac{\partial^{2} \theta}{\partial X^{2}}+\frac{\partial^{2} \theta}{\partial Y^{2}}+\frac{\partial^{2} \theta}{\partial Z^{2}}\right)
\end{aligned}
$$

The non-dimensional variables are defined as follows:

$$
\begin{gathered}
X=\frac{x}{L}, Y=\frac{y}{L}, Z=\frac{z}{L} \\
U=\frac{u}{u_{i}}, V=\frac{v}{u_{i}}, W=\frac{w}{u_{i}}, P=\frac{p-p_{0}}{\rho_{0} \cdot u_{i}{ }^{2}}, \theta=\frac{T-T_{c}}{T_{h}-T_{c}}
\end{gathered}
$$

Where $p_{0}$ and $\rho_{0}$ are respectively the reference pressure and density. $T_{h}$ is the temperature at the heated surface, $T_{c}$ the temperature at the cooled surface, $p$ is the pressure and $(\mathrm{U}, \mathrm{V}, \mathrm{W})$ are the velocity components.

In the above equations, the parameters Pr, Gr and Re denote the Prandtl number, Grashof number and the Reynold number, respectively. These parameters are defined as:

$$
R e=\frac{L \cdot u_{i}}{v}, G r=\frac{g b L^{3}\left(T_{h}-T_{c}\right)}{v^{2}}, R i=\frac{G r}{R e^{2}}, \operatorname{Pr}=\frac{v}{\alpha}
$$

Where $\beta, v$ and $\alpha$ are the thermal expansion coefficient, the kinematic viscosity and the thermal diffusivity, respectively.

The boundary conditions, associated to the problem are:

- $U=V=W=0$ on the rigid walls of the enclosure;

- $U=1, V=W=0, \theta_{c}=0$ at the inlet;

- $\theta_{\mathrm{h}}=1$ on the left vertical heated sections; $\frac{\partial \theta}{\partial \mathrm{n}}=0$ elsewhere on the wall

- $\quad \theta_{\mathrm{c}}=0$ on the right vertical coold wall;

- $\frac{\partial \theta}{\partial \mathrm{n}}=0$ on other vertical and horizontal walls and the right and left sections near the opening (" $n$ " is the normal direction to the considered wall);

- $\frac{\partial \mathrm{U}}{\partial \mathrm{X}}=0, \frac{\partial \theta}{\partial \mathrm{X}}=0, \mathrm{~V}=\mathrm{W}=0$ at the outlet.

The local Nusselt number and the average Nusselt characterizing the heat transfer at the heated walls are respectively defined by:

$$
\mathrm{Nu}_{\text {local }}(\mathrm{Y}, \mathrm{Z})=\frac{\partial \theta}{\partial \mathrm{X}} \quad \text { at } \quad \mathrm{X}=0
$$




$$
\mathrm{Nu}_{\mathrm{h}}=\frac{2}{\varepsilon^{2}} \int_{\frac{\mathrm{L}-\varepsilon}{2} \frac{\mathrm{L}+\varepsilon}{2}}^{\frac{\mathrm{L}+\varepsilon}{4}} \int_{\frac{\mathrm{L}}{4}}^{4} \mathrm{Nu}(\mathrm{Y}, \mathrm{Z}) \mathrm{dYdZ}+\frac{2}{\varepsilon^{2}} \int_{\frac{\mathrm{L}-\varepsilon}{2}}^{\frac{\mathrm{L}+\varepsilon}{2}} \int_{\frac{3 \mathrm{~L}-\varepsilon}{4}}^{\frac{3 \mathrm{~L}+\varepsilon}{4}} \mathrm{Nu}(\mathrm{Y}, \mathrm{Z}) \mathrm{dY} \mathrm{dZ}
$$

\section{Numerical method}

The incompressible Navier-Stokes and energy equations are discretized by the finite volume method developed by Patankar [6] adopting the power law scheme for the convective terms. The SIMPLEC algorithm is used to couple momentum and continuity equations. The discretized equations are iteratively solved using an Alternating Direction Implicit (ADI) scheme. The system of algebraic equations is solved iteratively by means of the Thomas algorithm. Convergence of the numerical code is established according to the following criterion:

$$
\sum_{\mathrm{i}, \mathrm{j}, \mathrm{k}=1}^{\operatorname{imax}, \mathrm{mmax}, \mathrm{kmax}} \frac{\left|\phi_{\mathrm{i}, \mathrm{j}, \mathrm{k}}^{\mathrm{n}+1}-\phi_{\mathrm{i}, \mathrm{j}, \mathrm{k}}^{\mathrm{n}}\right|}{\left|\phi_{\mathrm{i}, \mathrm{j}, \mathrm{k}}^{\mathrm{n}}\right|} \leq 10^{-4}
$$

Where $\phi$ represents a dependent variable $U, V, W, T$, and $P$, the indices $i, j$, and $k$ indicate the grid positions, $\mathrm{n}$ represents the iteration number.

The present computational code, used to solve the governing equations, was validated after a comparison between our results and those presented by Moraga \& López [5] in terms of temperature distribution and velocity profiles presented in Fig $2 \mathrm{a}$ and Fig $2 \mathrm{~b}$ respectively. Hence, a good agreement was found with the Three-dimensional results of Moraga \& López [5]. Uniform grid sensitivity tests were also conducted for different sets of the governing parameters and showed that the grid size $91 \times 71 \times 71$ was the most appropriate for the present study.
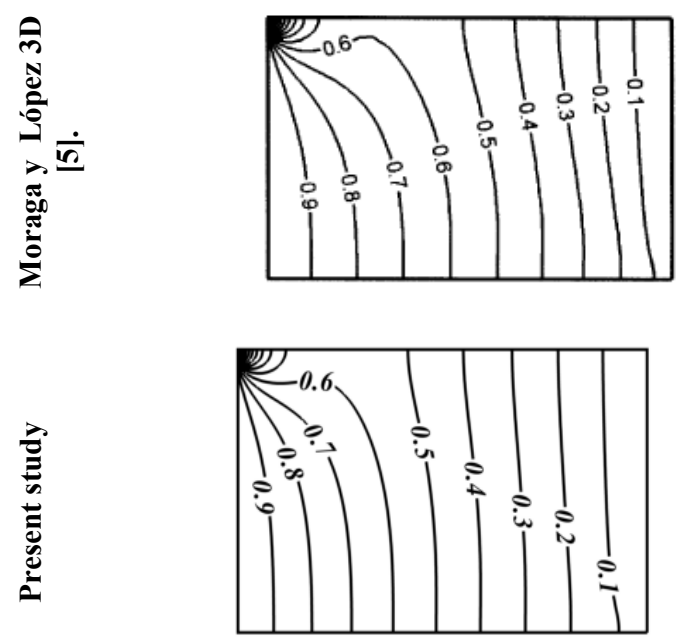
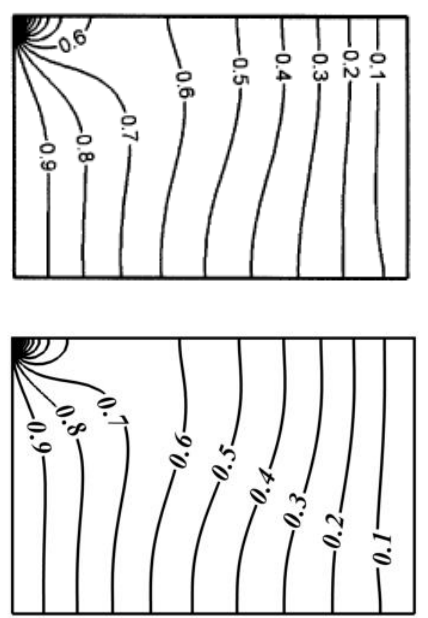

Fig. 2a. Comparison between the isotherm obtained at the middle plane $Z=0.5$ and those of Moraga and López [5]. 
a)

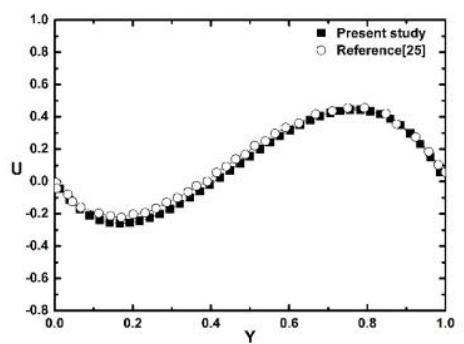

b)

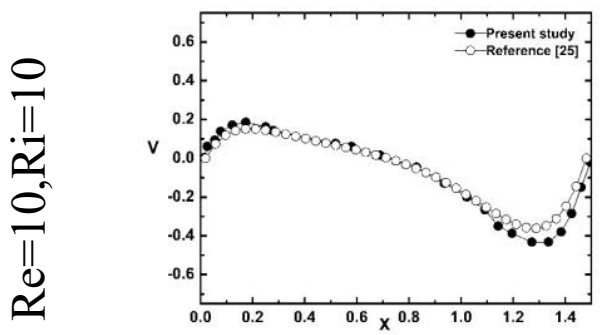

Fig. 2b. $U$ velocity component at $X=0.75, Z=0.5$ and b). $V$ velocity component at $Y=0.5, Z=0.5$

\section{Results and discussion}

The aim of this paper is to study the effects of the Richardson and Reynolds numbers on the thermal transport and fluid flow phenomena in the cavity. The numerical computations are conducted for a wide range of Richardson number $0 \leq \mathrm{Ri} \leq 10$, Reynolds number $50 \leq \operatorname{Re} \leq 100$, which correspond to a laminar regime. The relative height of the opening $\mathrm{B}=\mathrm{h} / \mathrm{L}$, the Prandtl number, the relatives height of the heated portions $\varepsilon=\mathrm{D} / \mathrm{L}$ and their width $\varepsilon / 2$ are maintained respectively at constant values: $1 / 8,0.71,0.3$ and 0.15 .

\subsection{Characteristics of the flow and the thermal fields}

In order to give a global overview of the cavity, 3D streamlines and isotherms are respectively shown in figures $3 \mathrm{a}$ and $3 \mathrm{~b}$, for $\mathrm{Re}=50$ and $\mathrm{Ri}=1$. It is seen that the fluid flow consists of a clockwise rotating cell which appear inside the cavity under the open lines of the forced flow. These last ones are directed to the outlet. The interaction between the incoming jet flow and the heated portions leads to important changes in the temperature distribution through the cavity toward the cooled wall as seen in the corresponding isotherm.

a)

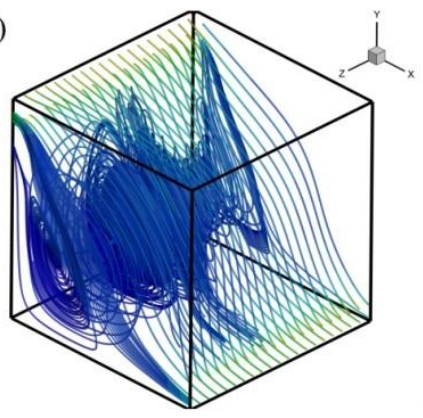

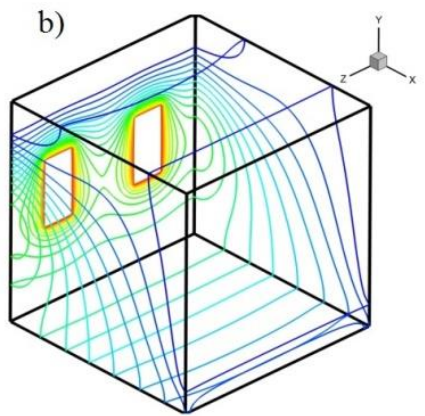

Fig. 3. 3-D streamlines (a) and isotherm (b) for $\mathrm{Re}=50$ and $\mathrm{Ri}=1$.

The effects of the Reynolds and Richardson numbers on the flow structure at the plane $\mathrm{Z}=0.5$ and temperature distribution are shown in Fig. $4 \mathrm{a}-\mathrm{b}$. In fact, a previous analysis of the isotherms and streamlines in different plans $(0 \leq Z \leq 1)$ shows a perfect symmetry with respect to the plane $Z=0.5$. This is due to the geometrical symmetry and adopted thermal 
boundary conditions. Hence, the planes $\mathrm{Z}=0.5, \mathrm{X}=0.025$ and $\mathrm{Y}=0.5$ were chosen for the following presentations. Indeed, these plans are characterized by higher activity and presented adequately the dynamical and thermal fields within the cavity.

The results show the existence of three kinds of heat transfer regimes, which depends on the buoyancy parameter Ri values. The first structure is shown in Fig. 4 for $\mathrm{Ri}=0$ and low Reynolds numbers $(\mathrm{Re}=50)$. In this case, the inflow is not strong enough, the open lines pattern conserves the same form and occupy almost the whole cavity until the outlet. A small vortex is located beneath the open lines of the forced flow. Furthermore, the size of the vortex increases by increasing Re to 100 . Due to this increased inertia force, the corresponding isotherms are stratified over the two heating sections due to the imposed boundary conditions while the lower isotherms are uniform and parallel to each other in the remaining parts of the cavity which signify a dominant conduction heat transfer.

At higher Re values, $R e=200$, the flow structure become intense and the intensity of the forced flow the open lines and the vortex size increases. Moreover, a secondary counter clockwise vortex appears in the upper corner of the cavity. The examination of the isotherm lines shows an improvement in the exchange at the cold wall of the cavity.

The higher temperature regions are concentrated near the heated portions while the thermal gradient increases and are submitted to a slight deflection. Indeed, pointing out that vigorous actions of inertia forces are the main role of the flow transport.

a)
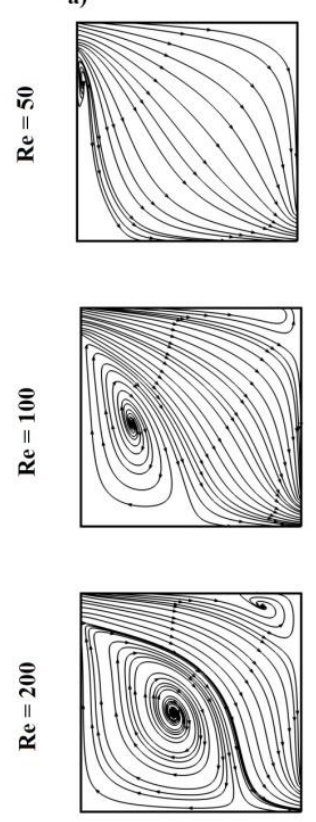

b)
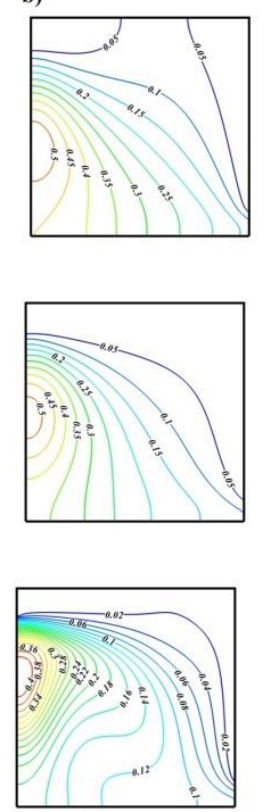

c)
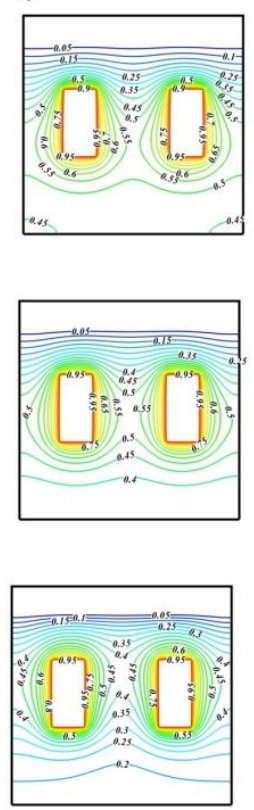

d)
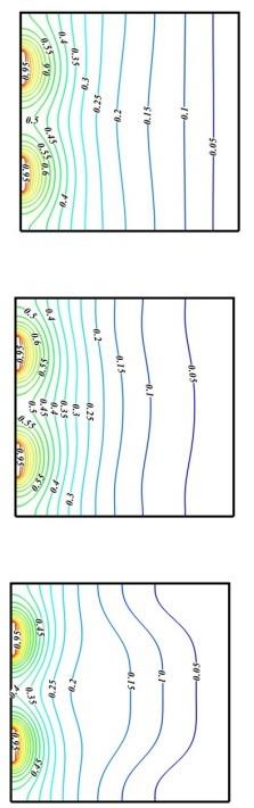

Fig. 4. Streamlines (a) and isotherms (b) at the mid-plane $X Y(Z=0.5)$, isotherms (c) at the plane $Y Z$ $(\mathrm{X}=0.025)$ and isotherms at the mid-plane $\mathrm{XZ}(\mathrm{Y}=0.5)$ for $\mathrm{Ri}=0$ and different values of Re.

The second structure is shown in Fig. 5 in terms of dynamical and thermal fields for $\mathrm{Ri}=1$ and different Re. The behaviour of convective heat transfer is the same as discussed previously in Fig 3 on 3D-model, in the case of low Reynolds number. However, the circulation in the flow becomes larger and the size of the vortex increases by increasing Reynolds number. The corresponding isotherms are more tightened at the vicinity of the heated portions and the gradient temperature becomes larger due to thermal interaction between the heated portions and the incoming cold flow which ensure well the installation of mixed convection. 
At higher value of Richardson number $(\mathrm{Ri}=10)$, the role of the free convection in the cavity becomes more significant (Fig. 6) and consequently the increase of all considered Reynolds numbers is accompanied by a reduction of the convective cell. This can be attributed to the dominance of thermal buoyancy effect where the boundary layers become

a)
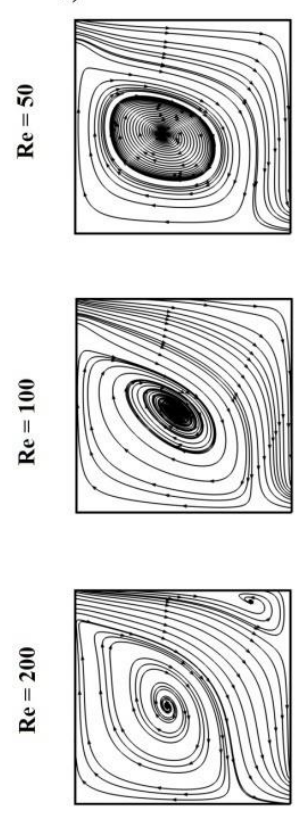

b)
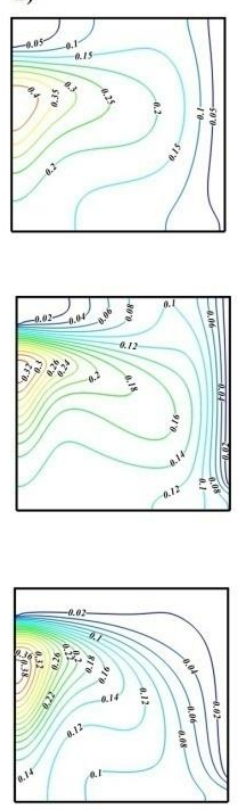

c)
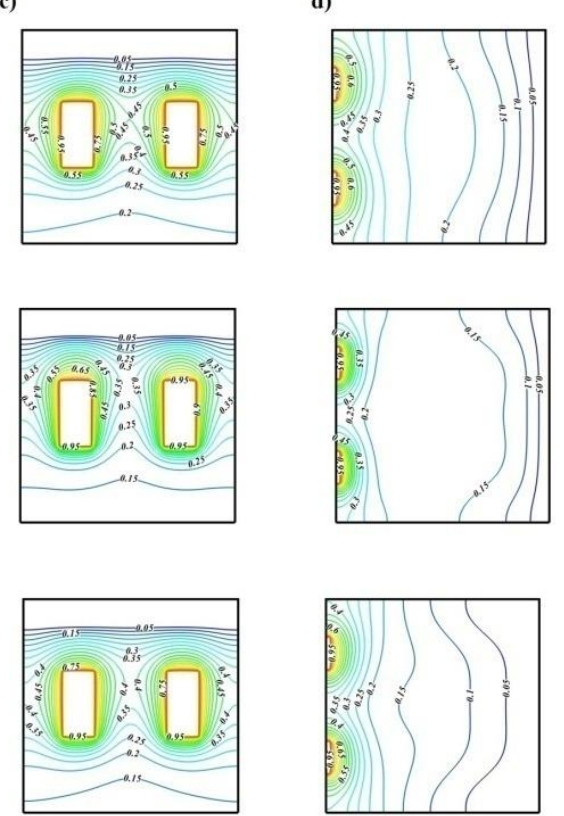

d)
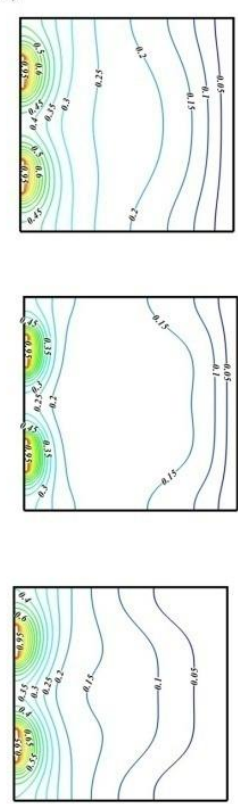

Fig. 5. Streamlines (a) and isotherms (b) at the mid-plane $X Y(Z=0.5)$, isotherms (c) at the plane $Y Z$ $(\mathrm{X}=0.025)$ and isotherms at the mid-plane $\mathrm{XZ}(\mathrm{Y}=0.5)$ for $\mathrm{Ri}=1$ and different values of Re. 
a)
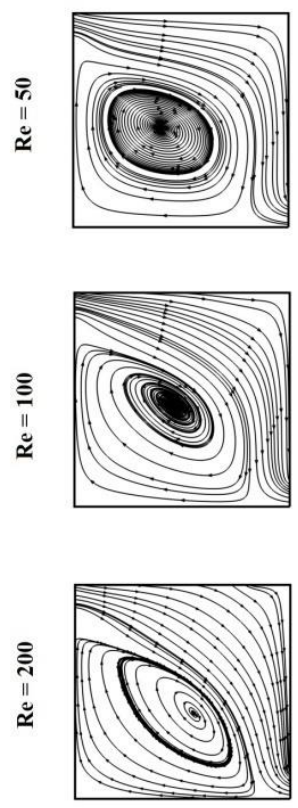

b)
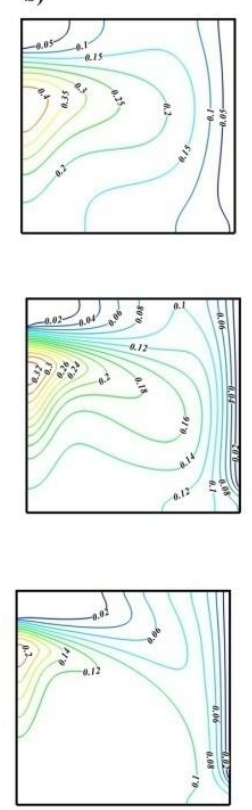

c)
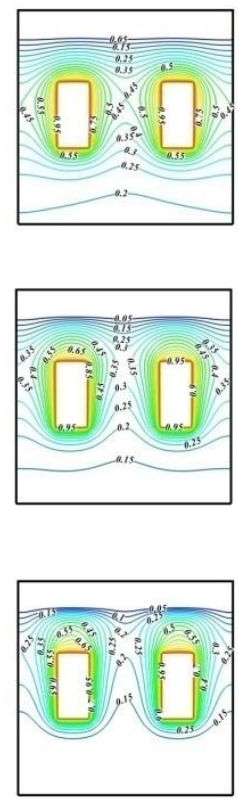

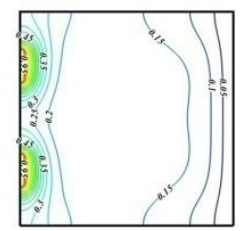

d)
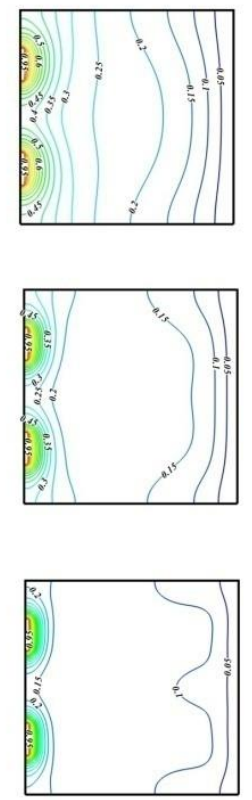

Fig. 6. Streamlines (a) and isotherms (b) at the mid-plane $X Y(Z=0.5)$, isotherms (c) at the plane $Y Z$ $(\mathrm{X}=0.025)$ and isotherms at the mid-plane $\mathrm{XZ}(\mathrm{Y}=0.5)$ for $\mathrm{Ri}=10$ and different values of Re.

\subsection{Heat transfer enhancement}

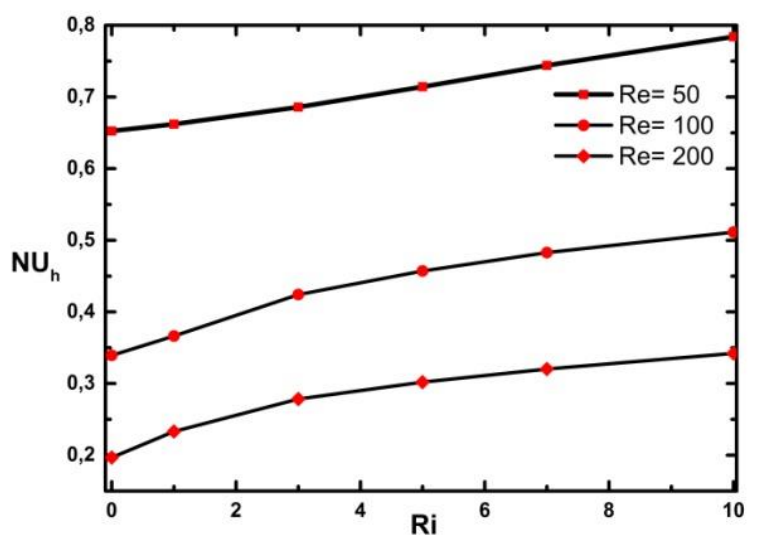

Fig. 7. Variation of the total average Nusselt number with Ri for different Re.

In order to analyze the thermal performance in our configuration, we illustrate in Fig. 7, the total average Nusselt number evaluated on the two heating sections versus $\mathrm{Ri}$, for different value of Re. As expected, the total average Nusselt number increases with the Richardson number. This can be explained by the fact that the buoyancy effects are the dominant mode of mixed convection transport. In contrast, for a given number $\mathrm{Ri}$, the 
Nusselt number decreases by increasing Re. This is due to the acceleration of the cold forced flow through the cavity along the cold wall which does not mix well with the internal heated air.

\section{Conclusion}

A numerical study has been carried out in the case of laminar three-dimensional mixed convection discretely heated from the side. The results show that the controlling parameters $\mathrm{Re}$ and $\mathrm{Ri}$ leads to important modifications of dynamical and thermal structures. The increase of the Richardson numbers enhances significantly the heat transfer. however, the forced flow support the heat transfer for low Reynolds numbers and plays an opposing role for the high values of Re.

\section{References}

1. E. Papanicolaou, Y. Jaluria, Numer, Heat. Tr. 18, 4, 427-461 (1991)

2. E. Papanicolaou, Y. Jaluria, Numer, Heat. Tr. 23, 4, 463-484. (1993)

3. T.H. Hsu, P.T. Hsu, S.P. How, Numer, Heat. Tr. 31, 6, 655-683 (1997)

4. M.M. Rahman, S. Parvin, N.A. Rahim, M.R. Islam, R. Saidur, M. Hasanuzzaman, Appl. Math. Model 36, 5, 2056-2066 (2012)

5. N.O. Moraga, S.E. Lopez, Numer, Heat. Tr. 45, 8, 811-824 (2004)

6. S. Patankar, CRC press (1980) 